
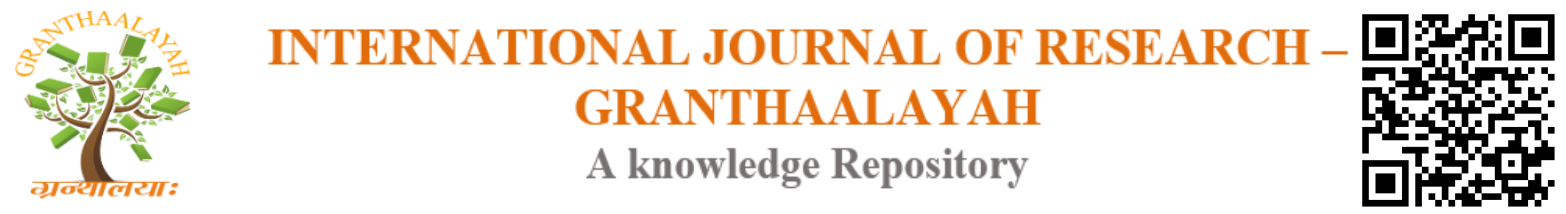

Science

\title{
SPATIAL AUTOCORRELATION ANALYSIS OF CITY PM2.5 CONCENTRATION IN HENAN
}

\author{
Hongling Meng *1, Kaiguang Zhang ${ }^{2}$, Mingting Ba ${ }^{2}$, Yanmin Sun ${ }^{2}$ \\ ${ }^{*}$ School of Mathematics and Statistics, Zhengzhou Normal University, China \\ ${ }^{2}$ School of Geography and Tourism, Zhengzhou Normal University, China
}

\begin{abstract}
PM2.5 has become the main pollutant of air pollution in China, and PM2.5 pollution control is one of the important means of atmospheric environmental governance. Aiming on the spatial and temporal distribution characteristics of regional PM2.5 concentration, this paper bases on the monitoring data of city PM2.5 concentration in Henan Province from 2015 to 2018 to study the spatial autocorrelation characteristics of city PM2.5 concentration and explore the city PM2.5 concentration spatial relationship by using the geo-statistical analysis method. The results showed that: the PM2.5 concentration in Henan shows obvious seasonal variation characteristics, the PM2.5 concentrations in the northern cities are significantly higher than that in the southern cities. The PM2.5 concentrations in the northern cities vary significantly with the seasonal transition, PM2.5 pollution is mainly moderately and above polluted, the PM2.5 concentrations in southern cities vary little with the seasonal transition, PM2.5 pollution is mainly lightly polluted. The city PM2.5 concentration shows a trend of regional integration with the significant spatial autocorrelation, the global autocorrelation characteristic is independent of PM2.5 concentration. The city PM2.5 concentration also presents the characteristics of local instability, forms a HighHigh aggregation region centered on Xinxiang and Zhengzhou, the aggregation degree tends to be significant with the increase of PM2.5 concentration, the aggregation area gradually expands to northward. The PM2.5 concentration in Anyang is no significant autocorrelation with the PM2.5 concentrations in other cities of the province.
\end{abstract}

Keywords: PM 2.5 Concentration; Seasonal Evolution; Global Spatial Autocorrelation Analysis; Local Spatial Autocorrelation Analysis; Spatial Aggregation; Henan Province.

Cite This Article: Hongling Meng, Kaiguang Zhang, Mingting Ba, and Yanmin Sun. (2019). "SPATIAL AUTOCORRELATION ANALYSIS OF CITY PM2.5 CONCENTRATION IN HENAN." International Journal of Research - Granthaalayah, 7(8), 454-462. https://doi.org/10.29121/granthaalayah.v7.i8.2019.699.

\section{Introduction}

With the continuous development of China's economy, the advancement of urbanization and industrialization, the increase of energy and natural resources consumption, a large amount of 
harmful substances have been discharging into the atmosphere, which have been causing many serious ecological problems, especially atmospheric problem in many urbanized regions. Air pollution seriously has been affecting people's physical and mental health, and largely inhibiting the healthy development of the regional economy [1-3].

The components of air pollutants in China city mainly include PM2.5, PM10, SO2, NO2, CO and $\mathrm{O} 3[2,4]$. The statistical results show that PM2.5 is the primary pollutant in most cities in polluted weather, which causes more than $50 \%$ polluted days of the total pollution weather. PM2.5 is atmospheric particulate matter, mainly composed of water-soluble ions, carbon components and chemical elements, with aerodynamic equivalent diameter less than 2.5 microns, has the characteristics of small diameter, large surface area, strong activity, strong adsorb ability for toxic and harmful substances (for example, heavy metals, microorganisms, etc.), long residence time in the atmosphere and long transport distance [4-8].

For the great harmful effects of PM2.5 on human health and atmospheric environment quality, it has received more and more attention from experts and scholars. In recent years, the researchers have focused on the components analysis and diffusion model analysis, the spatial distribution characteristics analysis in multi-scale of geoscience vision, and the influencing factors analysis, have been achieved a lot of useful results [4-13]. In fact, although the amount of PM2.5 emissions is the main factor determining regional concentration, the regional topographic conditions, wind direction, wind speed, precipitation and temperature have some impacts on the its spread, regional transmission and transport is one of its important features, the concentration in a region is affected by the concentrations of its adjacent regions, and there is some certain correlation between regions [3-10].

Bases on the monitoring data of city PM2.5 concentration in Henan Province from 2015 to 2018, this paper study the spatial autocorrelation characteristics of city PM2.5 concentration to explore its spatial relationship, by using the geo-statistical analysis method, in order to provide some scientific reference for the regional PM2.5 pollution control.

\section{Data and Research Methods}

\subsection{Research Area Overview and Data}

Henan province $\left(31^{\circ} 23^{\prime} \mathrm{N}-36^{\circ} 22^{\prime} \mathrm{N}, 110^{\circ} 21^{\prime} \mathrm{E}-116^{\circ} 39^{\prime} \mathrm{E}\right)$ is located in the central part of China, consists of 17 cities with a total area of 167 thousand $\mathrm{km}^{2}$, its three sides as the north, west and south are semi-circular surrounded by Taihang, Funiu and Dabie mountains, its central and east regions are Huanghuaihai alluvial plain[14]. the data used in the study mainly includes the monitoring data of city PM2.5 concentration in 17 cities (from 2015.1.1-2018.12.30) derived from the key city air quality daily report data of the Ministry of Environmental Protection of the People's Republic of China (http://datacenter.mep.gov.cn), and the city spatial distribution provided by Computer Network Information Center(http://datacenter.mep.gov.cn/). 


\subsection{PM2.5 Concentration Spatial Autocorrelation Model}

\subsubsection{Concentration Global Spatial Autocorrelation Model}

Affected by the natural conditions such as topographic conditions, atmospheric circulation, wind direction and wind speed, PM2.5 have the important characteristics of regional transmission and transportation. The PM2.5 concentration in a city is affected by the concentration in its adjacent cities, there are some certain correlations among them, these correlations decrease as the distance increases. Studying the overall distribution characteristics of PM2.5 concentration, could accurately understand its overall change characteristics and spatial similarity, and reveal the influence relationship among regional city agglomeration [12-14].

The global spatial autocorrelation analysis uses the test statistic Global Moran's I, defined as:

$$
I=\frac{\sum_{i=1}^{n} \sum_{j=1}^{n} w_{i j}\left(x_{i}-\bar{x}\right)\left(x_{j}-\bar{x}\right)}{S^{2} \sum_{i=1}^{n} \sum_{j \neq i}^{n} w_{i j}},
$$

where $x_{i}$ is the PM2.5 concentration in city $i,\left(w_{i j}\right)$ is the spatial relation weight matrix, $w_{i j}=1$ if city $i$ and $j$ have more than one common border, otherwise $w_{i j}=0, \bar{x}$ and $S^{2}$ respectively are the mean and the variance of PM2.5 concentration in the study region.

The value range of Global Moran's I belongs to $[-1,1], I>0$ indicates that the PM2.5 concentration is aggregated, most of cities and their adjacent cities have the same difference direction with the PM2.5 concentration mean in the study region. The larger $I$ means the more obvious aggregating tendency, the greater interacting of PM2.5 concentration among cities. $I<0$ indicates that the PM2.5 concentration is dispersed, some of cities and their adjacent cities have the different variety direction with the PM2.5 concentration mean in the study region. $I=0$ means that the PM2.5 concentration in the region is randomly distributed, no autocorrelation existing among them. Assessing the autocorrelation using the standardized PM2.5 concentration.

$$
Z=\frac{I-E(\mathrm{I})}{\sqrt{\operatorname{VAR}(I)}}
$$

as the test statistic, where $E(I), V A R(I)$ respectively are the mean and the variance of $I$, calculated by the number of cities and the spatial relation weight matrix $\left(w_{i j}\right)$, under the hypothesis of $I$ is a normal distribution.

\subsubsection{PM2.5 Concentration Local Spatial Autocorrelation Model}

Global Moran's I is a global assessment of spatial autocorrelation, ignoring the potential instability of spatial distribution. Local autocorrelation index is used to measure the influence degree of local spatial units to the overall spatial autocorrelation in the study region, and to what extent the global assessment of spatial autocorrelation masks abnormal local regions or small local instability [1214], defined as 
$I_{i}=\frac{\left(x_{i}-\bar{x}\right) \sum_{j \neq i}^{n} w_{i j}\left(x_{j}-\bar{x}\right)}{S^{2}}$,

$I_{i}$ describes the spatial aggregation degree between city $i$ and its adjacent cities with significant similar values.

Obviously, there is

$$
I=\frac{\sum_{i=1}^{n} I_{i}}{\sum_{i=1}^{n} \sum_{j \neq i}^{n} w_{i j}} .
$$

The local spatial autocorrelation index decomposes the global spatial autocorrelation into the contributions of each city, $I_{i}>0$ means city $i$ have the same deviation direction with most of its adjacent cities about the PM2.5 concentration with the PM2.5 concentration mean in the study region, the local region with $i$ center presents a high-high or low-low aggregation feature. $I_{i}<0$ means city $i$ have the different deviation direction with most of its adjacent cities about the PM2.5 concentration with the PM2.5 concentration mean in the study region, the local region for $i$ center presents low-value city $i$ surrounded by high-value cities(low-high) or high-value city $i$ surrounded by low-value cities, the local region with $i$ center presents some decentralization feature.

\section{Spatial Autocorrelation Analysis of City Pm2.5 Concentration in Henan}

\subsection{Statistical Distribution Characteristics Analysis of City Pm2.5 Concentration in Henan}

Among the 1452 valid samples from 2015 to 2018 in Henan, the air quality is mainly lightly polluted and above, the number of pollution days is about 1079 days, accounting for $74.31 \%$. PM2.5 is the main air pollution source, the number of days PM2.5 concentration above the minimum concentration of lightly polluted is about 866 days, accounting for $59.64 \%$ of the total days and $80.26 \%$ of the polluted days.

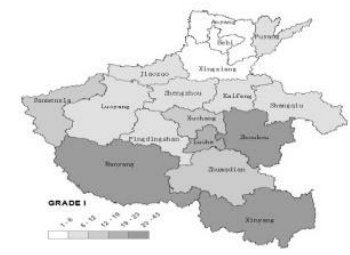

(a) Good days

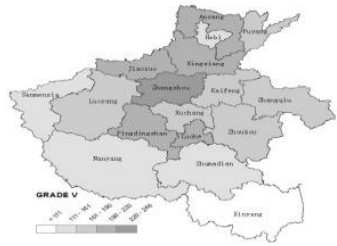

(e) Heavily polluted days (f) Heavily polluted days

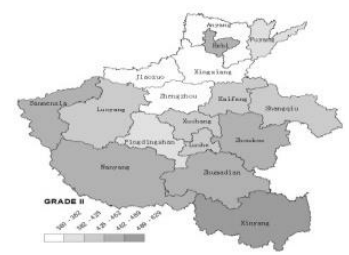

Moderate days

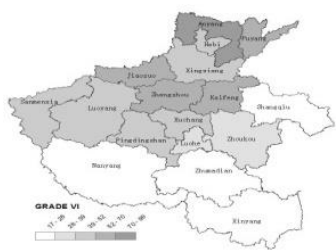

c) Lightly polluted days

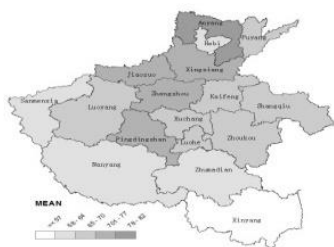

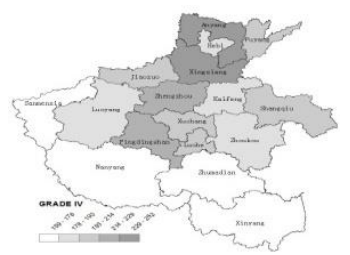

(d) Moderately polluted days

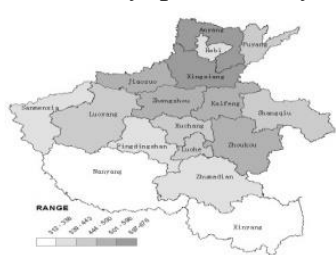

(h) Concentration range

Figure 1: The spatial distribution of city PM2.5 concentration in Henan (2015 to 2018) 
In the spatial distribution, the distribution of PM2.5 concentration between cities are quite different, the proportion of PM2.5 pollution days shows obvious regional characteristics, the cities in central and northern are significantly higher than those in southern, Anyang is highest up to $74.93 \%$, followed by Zhengzhou, Xinxiang, Jiaozuo, Puyang and Pingdingshan, all of which account for more than $70 \%$ (Fig. 1(a) and 1(b)).

There is a significant difference in the distribution of PM2.5 lightly polluted days and total PM2.5 polluted days, the proportion in the southern cities as Xinyang, Nanyang and Zhumadian accounts for more than $62 \%$ (Fig. 1(c)). The distribution of PM2.5 of moderately polluted days is similar with the distribution of total polluted days, the proportion of PM2.5 moderately polluted days to total PM2.5 polluted days is about $20 \%$ (Fig. 1(d)). The proportion of PM2.5 heavily polluted days to total PM2.5 polluted days is about $20 \%$ (Fig. 1(e)). The proportions of PM2.5 severely polluted days to total PM2.5 polluted days in Zhengzhou and Pingdingshan, cities in the central part of the province, account for more than $22 \%$, but the proportion in Xinyang, the city in the southern part, is less15\%(Fig. 1(f)).

During the study period, the polluted days is proportional to the PM2.5 concentration mean (Fig. $1(\mathrm{~g})$ ), but the PM2.5 concentration ranges presents some differentiation feature in the central region, especially in Pingdingshan and Zhoukou, their ranges respectively are 392 and 543 with the means of 73 and 67, only less than 676 in Xinxiang, 664 in Anyang and 596 in Zhengzhou(Fig. $1(\mathrm{~h}))$.

In general, the PM2.5 concentrations in the central and northern cities are significantly higher than that in the southern cities. The change of PM2.5 concentration in the northern cities is obviously, the pollution is mainly moderately polluted or above, and the change of PM2.5 concentration in southern cities is smaller, the pollution is mainly lightly polluted.

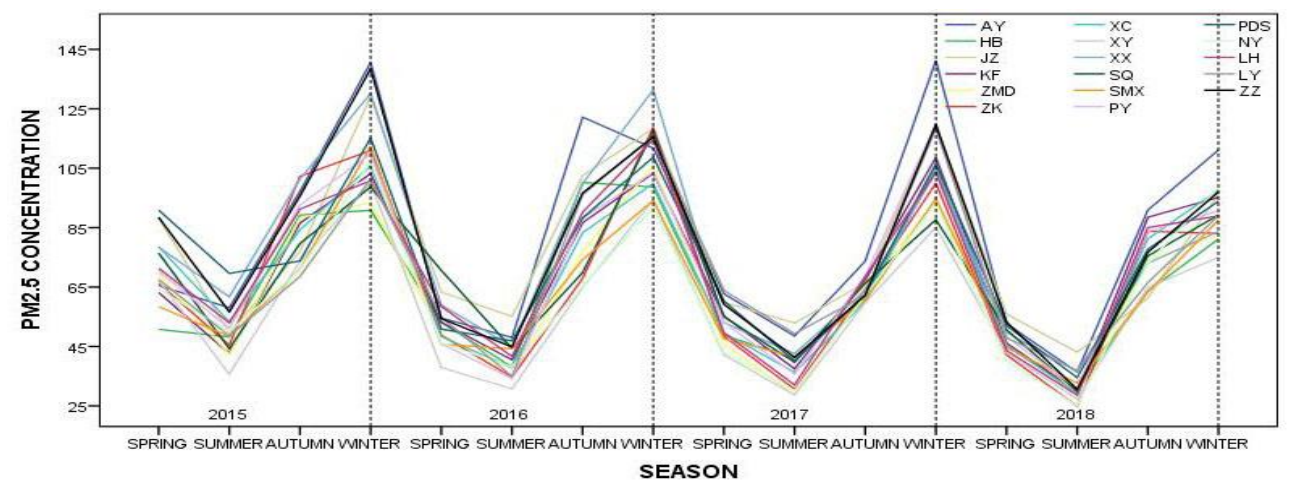

Figure 2: The seasonal distribution of city PM2.5 concentration in Henan (2015 to 2018)

\subsection{Temporal Distribution Characteristics Analysis of City Pm2.5 Concentration in Henan}

The annual mean of city PM2.5 concentration gradually decreases from 2015 to 2018, in a year, the city PM2.5 concentration shows obvious seasonal variation characteristics in all cities, and the seasonal variation characteristics are basically the same (Fig. 2), from the spring to the summer, the seasonal mean of city PM2.5 concentration gradually decreases, reaches the lowest value of the whole year, then gradually increases, reaches the maximum value in the winter. The order of 
the four seasons from low to high is summer, spring, autumn and winter. There are obvious differences in the change ratios of PM2.5 concentration with seasonal transitions, the absolute decline of PM2.5 concentration from winter to spring is greater than the increase of other seasonal transitions.

\subsection{Spatial Autocorrelation Analysis of City Pm2.5 Concentration in Henan}

The city PM2.5 concentration shows the same seasonal variation characteristics, the spatial autocorrelation of PM2.5 concentration seasonal mean could reflect the distribution characteristics and evolution of the whole region to a certain extent.

\subsubsection{Global Spatial Autocorrelation Analysis of City PM2.5 Concentration in Henan.}

Using Formula 1 and Formula 2 to calculate the global spatial autocorrelation indices and Z values for the four seasons (Spring, Summer, Autumn and Winter), the results are shown in Fig. 3. Global Moran's I of the four seasons respectively are $0.1882,0.2957,0.4590$ and 0.2069 , and the corresponding $\mathrm{Z}$ values respectively are 1.6119, 2.2873, 3.354 and 1.6979 , indicating that the city PM2.5 concentration shows certain positive autocorrelations under the significance level 0.05 in all the seasons. The autocorrelation in autumn is the strongest, followed by summer, winter and autumn. The autocorrelation show significant differences with and seasonal mean. From spring to autumn, the autocorrelation gradually increases, while the city PM2.5 concentration mean goes through a up-parabola process. The winter city PM2.5 concentration is the highest, but the autocorrelation is only between spring and summer.

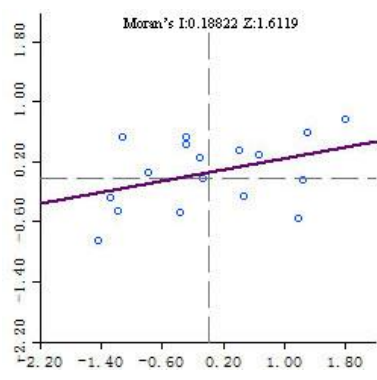

(a) Spring

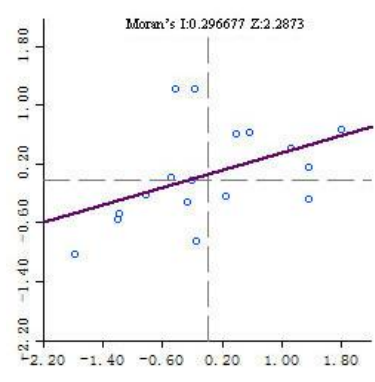

(b) Summer

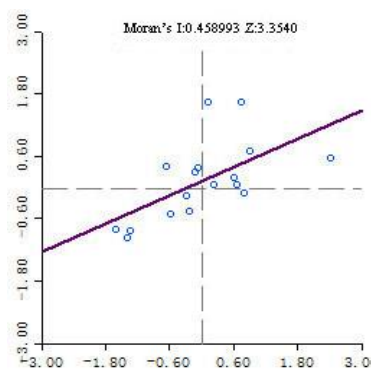

(d) Autumn

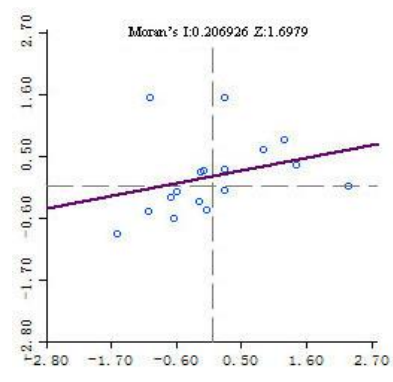

(c) Winter

Figure 3: The global spatial autocorrelation analysis of city PM2.5 concentration in Henan (2015-2018)

\subsubsection{Local Autocorrelation Analysis of City PM2.5 Concentration in Henan.}

In order to study the local instability in spatial distribution of city PM2.5 concentration, using Formula 3 to calculate the local autocorrelation index of city PM2.5 concentration in each season, the results are showed in Fig. 4, Fig. 5 is the distribution of PM2.5 concentration seasonal means. Spring (Fig. 4a,5a): The PM2.5 concentration means in all cities are less than the lightly polluted minimum (75), PM2.5 has less pollution to the atmospheric environment. The high-value areas have two regions, one is located in the central part of the province, including Jiaozuo (67), Zhengzhou (64), Pingdingshan (64), the other is Shangqiu (63) located in the eastern part of the province. Zhengzhou and Jiaozuo form a High-High aggregation hot-spot region, the other region have no significant aggregation characteristics. 


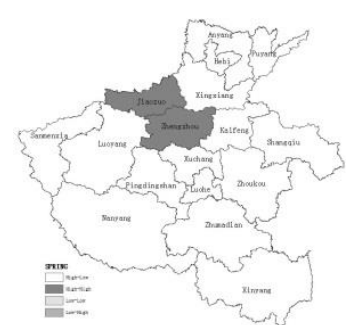

(a) Spring

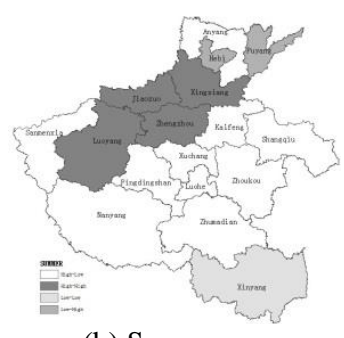

(b) Summer

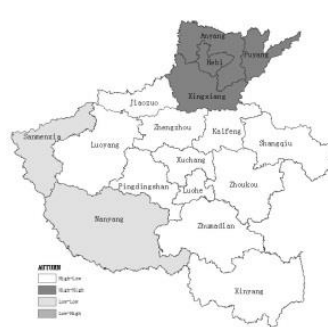

(c) Autumn

Figure 4: The local spatial autocorrelation analysis of city PM2.5 concentration in Henan (20152018)

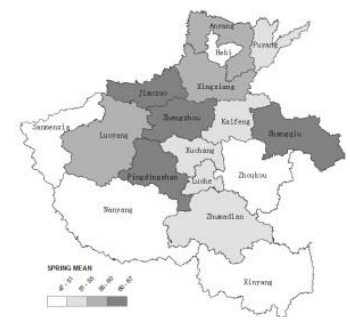

(a) Spring

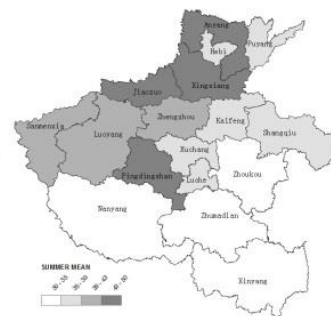

(b) Summer

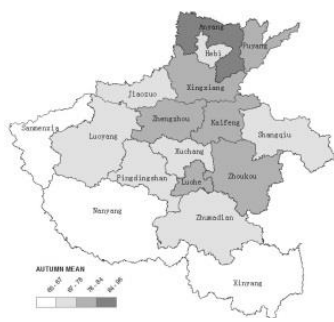

(c) Autumn

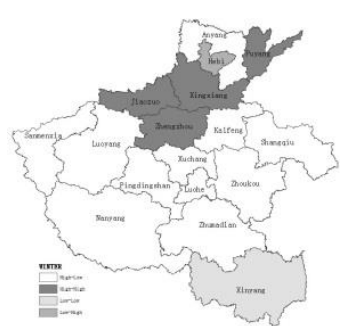

(d) Winter

Figure 5: The seasonal mean distribution of city PM2.5 concentration in Henan province (2015 to 2018)

Summer (Fig. 4b,5b): The PM2.5 concentration means in all cities is less than 50, PM2.5 has little pollution to the atmospheric environment. The high-value areas have two regions, one is located in the northern part of the province, including Jiaozuo (50), Anyang (48) and Xinxiang (46), the other is Pingdingshan (48) located in the central part of the province. Xinxiang, Zhengzhou, Jiaozuo form a High-High aggregation hot-spot region, Xinyang is a low-low aggregation coldspot region, Hebi and Puyang form a low-value heterogeneous region, surrounding by high value regions, and the other region have no significant aggregation characteristics.

Autumn(Fig. 4c,5c): The PM2.5 concentrations in all cities increase rapidly, the PM2.5 concentration means in Xinyang, Sanmenxia and Nanyang are less than the lightly polluted minimum (75), in other cities, the PM2.5 concentration means are obviously higher than the minimum. The northern cities, as Anyang, Hebi, Puyang, Xinxiang and Zhengzhou form a HighHigh aggregation hot-spot region, Nanyang and Sanmenxia form a strip low-low aggregation coldspot region along the southwestern part of the province, there is no significant aggregation characteristics in other regions.

Winter (Fig. 4d,5d): The PM2.5 concentrations in all cities reaches their maximum, PM2.5 is very polluting to the atmospheric environment. The PM2.5 concentration means in Anyang, Zhengzhou and Jiaozuo are all greater than the lightly polluted maximum, reaching moderately polluted level, PM2.5 pollution reaches lightly polluted level in other cities. Zhengzhou, Xinxiang, Jiaozuo and Puyang form a High-High aggregation hot-spot region, Xinyang is a low-low aggregation coldspot region, Hebi is a low-value heterogeneous region, surrounding by high value regions, and the other region have no significant aggregation characteristics. 
In general, the city PM2.5 concentration presents a trend of regional integration with the significant spatial autocorrelation, meanwhile in some regions also show the characteristics of local instability. Xinxiang and Zhengzhou form a local High-High aggregation region, its aggregation degree tends to be significant with the increase of PM2.5 concentration, the aggregation area gradually expands to northward, Xinyang is a local Low-Low aggregation region, The PM2.5 concentration in Anyang is no significant autocorrelation with the PM2.5 concentrations in other cities of the province.

\section{Conclusion}

Based on the monitoring data of city PM2.5 concentration in Henan Province from 2015 to 2018, this paper utilizes the geo-statistical analysis method to study the temporal distribution and spatial autocorrelation characteristics of city PM2.5 concentration and explore the city PM2.5 concentration spatial relationship. The results showed that: PM2.5 has been becoming the main atmosphere pollution source in the province, the city PM2.5 concentration shows obvious seasonal variation characteristics, winter is the season with the highest of PM2.5 concentration, followed by autumn, spring and summer. The PM2.5 concentration in the northern cities is significantly higher than that in the southern cities. The PM2.5 concentration in the northern cities varies significantly with the seasonal transition, PM2.5 pollution is mainly moderately and above polluted, the concentration of PM2.5 in southern cities varies little with the seasonal transition, PM2.5 pollution is mainly lightly polluted. The city PM2.5 concentration presents a trend of regional integration with the significant spatial autocorrelation, the global autocorrelation in autumn is obviously greater than that in other seasons, the global autocorrelation characteristic is independent of PM2.5 concentration. In some regions, the distribution of city PM2.5 concentration also shows the characteristics of local instability, gradually forms a high-high aggregation region centered on Xinxiang and Zhengzhou, the aggregation degree tends to be significant with the increase of PM2.5 concentration, the aggregation area gradually expands to northward. The PM2.5 concentration in Anyang is no significant autocorrelation with the PM2.5 concentrations in other cities of the province.

\section{References}

[1] Lin Xueqin, WANG Dai. Spatio-temporal variations and socio-economic driving forces of air quality in Chinese cities. Acta Geographica Sinica, Vol.71, No.8,2016, 1357-1371.

[2] Ma Z, Hu X, Sayer A, et al.2016. Satellite-based spatiotemporal trends in PM2.5concentrations: China, 2004-2013. Environmental Health Perspectives, No.124, 2016, 184-192.

[3] Liu H M, Fang C L, Zhang X L, et al.The effect of natural and anthropogenic factors on haze pollution in Chinese cities:A spatial econometrics approach. Journal of Cleaner Production, No.165,2017, 323-333.

[4] CHEN Liding, ZHOU Weiqi, HAN Lijian, et al. Developing key technologies for establishing ecological security patterns at the Beijing-Tianjin-Hebei urban megaregion. Acta Ecologica Sinica, Vol.36, No.22, 2016, 7125-7129.

[5] BIE Tong, HAN Lijian, TIAN Shufang, et al. Method for quantifying the contribution of urbanization on population exposure to air pollution. Acta Ecologica Sinica, Vol.38, No.13,2018, 4570-4583.

[6] WANG Guanlan, XUE Jianjun, ZHANG Jianzhong. Analysis of Spatial-temporal Distribution Characteristics and Main Cause of Air Pollution in Beijing-Tianjin-Hebei Region in 2014. Meteorological and Environmental Sciences, Vol.39, No.1,2016, 34 -42. 
[7] ZHANG Huitao, TIAN Yingze, LIU Baoshuang, et al. Spatial Temporal Characteristics and Cluster Analysis of Chemical Components for Ambient PM2.5 in Wuhan. Environmental Science, https://doi.org/10.13227/j.hjkx.201904069.

[8] YUAN Chang, ZHOU Jiabin, XIONG Ying, et al. Chemical Compositions and Long-range Transport of PM 2.5 in Downtown Area of Wuhan. Environmental Science \& Technology, Vol.41, No.8,2018, 79-86.

[9] Zhao X Y,Gu C,Yang H M, et al.. Chemical composition and source apportionment of PM 2.5 during a winter air pollution episode in the Kui DuWu Area of Xinjiang Uygur Autonomous Region. Research of Environmental Sciences, Vol.30, No.10, 2017, 1515-1523.

[10] ZHANG Kaiguang, BA Mingting, MENG Hongling et al. Spatial Distribution Characteristics and Evolution Pattern of Air Quality in Henan Province. Journal of Progressive Research in Mathematics (JPRM), Vol.15, No.1,2019, 2464-2468.

[11] Chen Y H,Wang Y J,Zhang M Y, Temporal and spatial distribution of PM 2. 5 in Shanghai based on clustering analysis. Chinese Journal of Environmental Engineering, Vol.11, No.6,2017, 36713677.

[12] Wen J, Shi X R, Tian Y Z, et al. Analysis of chemical composition of the fine particulate matter in summer in Tianjin City via a single particle aerosol mass spectrometer (SPAMS). Environmental Science, Vol.39, No.8,2018, 3492- 3501.

[13] Liu T, Wang X J, Chen Q, et al. Pollution characteristics and source apportionment of ambient PM2.5 during four seasons in Yantai City. Environmental Science, Vol.40, No.3,2019,1082-1090.

[14] ZHANG Kai-guang, BA Ming-ting, MENG Hong-ling et al. Spatial Correlation Analysis of Urban Air Quality in Henan Province. SCIREA Journal of Geosciences, Vol.3, No.1, 2019, 1-12.

[15] Zhang Kaiguang, Ba Mingting, Meng Hongling. Spatial pattern and forecast analysis of urbanization growth rates in Henan (2011-2015). AER-advance in engineering research, No.57,2016, 45-49.

[16] Chen Yanguang. Mathematical Methods for Geography. Beijing: Science Press,2017

*Corresponding author.

E-mail address: zzgis@ 126.com 